\title{
Virtual-spine
}

\section{The Collaboration Between Pervasive Environment Based Simulator, Game Engine (Mixed- Reality) and Pervasive Messaging}

\author{
Stephen Jia Wang \\ Department of Design \\ Faculty of Art Design \& Architecture, Monash University \\ Melbourne, Australia \\ Stephen.wang@monash.edu
}

\begin{abstract}
This paper outlines a project called "Virtual-spine" which aims to convert seated posture data into interactive 3D representations of spine movement. Spine movement information is collected from pressure sensors installed at various locations on the seat. Sensor readings are combined with "chair-id" and user "profile" to obtain contextual hypotheses. The output is delivered via a messaging system developed to help prevent harmful and fixed postures in prolonged sitting. A specific setup for evaluation testing is also outlined.
\end{abstract}

Keywords- spine; sitting; health; interactive $3 D$; pervasive computing; context; unity $3 \mathrm{D}$; arduino

\section{INTRODUCTION}

Sitting is one of the most common behaviors in people's daily life. A recent epidemiological study on about 50,000 adults from 20 countries reported sitting time was 300 minutes/day on average [1]. Incorrect sedentary positions and prolonged sitting has become a serious health threat to people in modern societies and various spinal problems. A large number of studies have convincingly reported the association between different levels of exposure to occupational sitting and the presence or severity of low back pain (LBP) [2]. There is also unequivocal evidence that sitting and upper quadrant musculoskeletal pain (UQMP) are related [3]. The third European Working Conditions Survey identified the most common work-related health problem as backache reported by $33 \%$ of respondents [4]. These problems often result in absence from work or even permanent disability and translate to high economic costs [5].

It is a challenge to maintain appropriate sitting positions in daily life to avoid seating related health issues. In the literature discomfort and pressure sores, have received particular attention in military [6], workplace [7] assisted living [8, 9] and mobility [10-12] contexts. For instance, the findings from Caneiro et al. demonstrate a clear link between thoraco-lumbar postures while sitting and head/neck posture and motor activity [13]. Burnett et al. articulate the challenge to maintain appropriate sedentary behavior to reduce extra burden to the spin and trunk muscles [14].

\section{SPINAL MOTION ANALYSIS}

What is good posture? According to Claus et al. [17] "Good posture may be influenced by demands to prevent movement, coordinate movement, safely load spinal segments

\author{
Di Yu \\ Department of Immunology \\ School of Biomedical Sciences, Monash University \\ Melbourne, Australia \\ di.yu@monash.edu
}

or conserve energy." Despite the controversy around what constitute an ideal sitting posture [15], it is clear that fixed postures, particularly in prolonged sitting, constitute a high risk factor for developing LBP due to static loading of soft tissues and discomfort [16]. As a preventive strategy, fixed postures in prolonged sitting should be avoided. Therefore, monitoring the spinal movements to prompt appropriate proactive measures is urgently needed.

Motivated by these findings, researchers from different disciplines are working on automatic monitoring of sitting postures to promote healthy sitting behavior. Specifically, clinicians are increasingly adopting spinal motion analysis as a useful clinical method to quantify the range of trunk motion and pattern of posture changes for diagnosis and outcome evaluation. For example, skin surface tracking (markers/sensors adhered to skin overlying spinous processes) has been used to quantify spinal curve and the change in lumbar spinal curve between positions from flexion to extension to identify optimized sitting posture [17]. Haller et al. equipped an ergonomic office chair with four sensors to measure an office worker's posture and to produce different alerts to help people improving their sitting posture [18]. However, the possibility of using a Pervasive Environment Simulator (PES) to collect and present through interactive 3D display detailed postural information has not been investigated.

Nowadays, with the development of intelligent embeddedagents and pervasive computing environments, performing experiments in a PES based game engine environment has become a cost-effective method to simulate users' real-time behavior [19]. The ideal model of a PES user monitoring and advisory system would comprise four seamlessly integrated modules a) multiple sensors, b) comprehensive data analysis (agents), c) interactive 3D (Mixed Reality), and d) messaging.

Such a system enables multiple users to simultaneously view, discuss, and interact with the virtual 3D models, and enhance practice by supporting remote and co-located activities [20]. The multiple sensors module monitors various data about the users. A comprehensive agent model is designed to constantly monitor users' everyday activities [21]. Interactive 3D (Augmented Reality or MR [22]) displays provides great flexibility of viewpoint and intuitive interfaces to present information and support users to change their behavior [23]. Such a Messaging system may act as powerful 
persuader because it can intervene in the right context as a convenient way to prompt users to change their behavior [24, $25]$.

Ubiquitous computing and context-aware persuasive technologies [24] offer an opportunity to promote healthy behavior by presenting "just-in-time", "appropriate time" and "appropriate place" information [26, 27]. An interesting discovery is that the appropriate sedentary position varies depending on the purpose people sit for, e.g. sitting in the office, car, dining etc. The main focus of our system design is to monitor people's sedentary behavior across various circumstances and encourage people to maintain appropriate sedentary positions under various contexts. To give location-aware advice the system relies on the "chair id" which reflects the surrounding environment. To give advice at the right moment the system requires knowledge of the users' activities.

Advice on correct sedentary posture must fit easily into users' daily routine since messages suggesting simple activities are preferred over ones requiring significant effort [28]. Besides, lifestyle interventions can yield positive and long-term effects, in terms of increasing levels of moderately intense physical activity [29]. The suggested locations of lifestyle activities we include in our system are [29]: everyday activity (shops, homes, schools, workplaces, etc.) and recreation destinations (playgrounds, parks and gardens, etc.)

Based on the current research and technical capabilities outlined above, in the next sections, we present a "Virtual-spine" system that provides users with personalized and contextualized advice on appropriate sitting positions. We introduce the design, implementation and a planned evaluation test of the Virtual-spine system.

\section{IMPLEMENTATION}

Virtual-spine is implemented using the Unity3D and Arduino platforms. The Unity3D engine supports exporting application to mobile platforms, which will render the 3D images in real-time based on the data from the Sedentary Position Analysis Unit.

\section{A. System Architecture}

Virtual-spine consists of:

- Sedentary-Sensory Unit

- Advisory Unit

- Interactive 3D Unit

- $\quad$ Messaging Unit

The "Sedentary-Sensory Unit" is designed to monitor users' spine movements while sitting by detecting center of gravity and back curvature. This unit can be designed in suitable forms, such as pad-like shape, and set up in various places to gather sedentary position information in different contexts like at the office, at home or driving. Each unit has a unique id used to recognize which chair the user is sitting on. This information is sent to the "Advisory Unit" which processes it to compute the cumulative spinal burden. Comprehensive sedentary information is presented to the user on mobile devices, computer screens or smart TVs as real-time rendered interactive $3 \mathrm{D}$ images. To reduce the burden on spine and motivate users to maintain healthy sedentary habits, messages are presented pervasively using the most appropriate media. For instance, advice will be displayed on TV when the user is sitting on the sofa or on a mobile device if the user is sitting on an office chair. Presentation settings could be tailored to users' privacy and other needs. The system architecture is illustrated in Fig. 1.

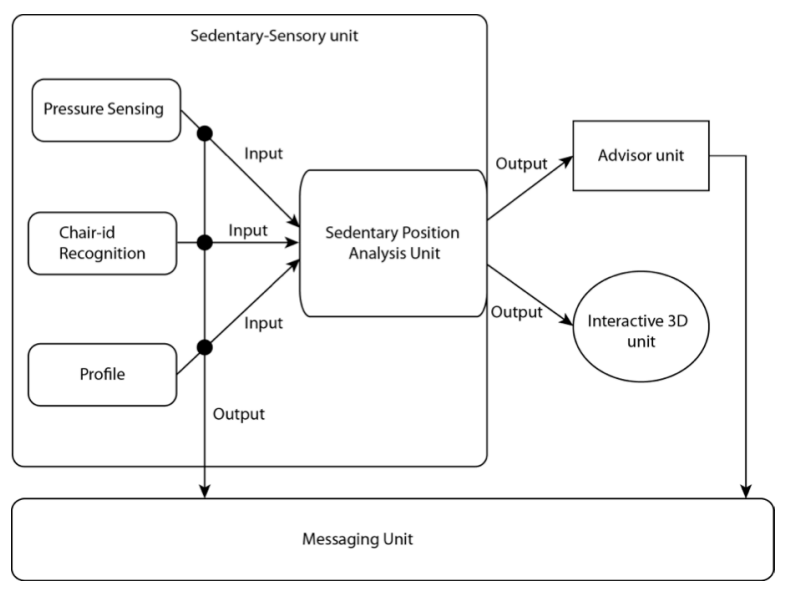

Figure 1. System Architecture

\section{B. Sedentary-Sensory Unit}

The "Sedentary-Sensory Unit" provides high-sensitivity "Pressure Sensing", "Chair-id Recognition" and "Profile" functions. When users sit or semi-sit on the sensing pad, their body affects the pressure sensors on it. Each sensor sends the detected values as analog input, which affects the $3 \mathrm{D}$ visualization through the "Advisory Unit". Multiple physical pads should be placed on the seats habitually used for the different purposes. The pads' ids determine the use context of each seat to provide correct posture parameters to the Sedentary Position Analysis unit. If the incorrect sedentary posture lasts over a set time threshold, the Advisor Service sends a message to the mobile UI.

\section{1) Pressure Sensing}

We used Force-Sensitive Resistor (FSR) sensors and an Arduino platform to detect postural changes. Sensors are laid out using a "near-optimal sensor placement strategy"[30]. Any curvature variation of the body trunk is converted to analog input that affects the virtual 3D spine in real-time. The connection between Arduino and Unity3D is provided by a "unity-arduino serial connection" [31]. Figure 2 shows the "Sedentary-Sensory Unit" (left), sensors affecting virtual mat (middle) and interactive 3D spine (right).

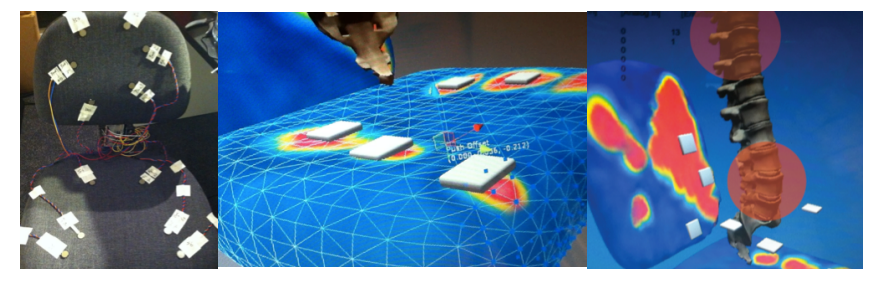


Figure 2. Sedentary Sensory Unit

\section{2) Chair-id Recognition}

Functionality and ergonomic characteristics such as seat height, depth, back support angle, surface material, flexibility (adjustment), etc. for each chair are stored in a database with a unique id. The "chair-id" is recognized when users sit and combined with users' profiles to calculate the most appropriate postures and sitting periods.

The Chair-id recognition function is based on Internet of Things technology. Currently, there are only a few companies providing free services to store ids, profiles and input/output data in real-time (pachube.com, open.sen.se, etc.) Chair specification could either be directly input or acquired from the manufactures' database. The latter option also creates opportunities to design future 'smart' chairs, which could monitor ergonomic adjustments and send them to the manufactures' database to inform design refinements. Users fill-in their profiles trough the online interface providing information such as body size, gender, age, common sitting time, type of transportation, etc.

\section{3) Sedentary Position Analysis Unit}

Unhealthy postures are recognized based on duration and detected deviations from the ideal spinal position. Claus, et al. [17] suggest, the 'ideal' sitting position depends on the angles of three divided spine sections: "thoracic, thoraco-lumbar and lumbar". The posture detection system uses a comparison algorithm to analyze the spinal positions of each section. The chair-seat sensors are divided into four sections: a (left-front), b (left-back), c (right-front) and d (right-back) to work out the position of the lumber area. The chair-back is divided into two sections: e (left) and $\mathrm{f}$ (right) to calculate the position of the thoracic area. The Analysis Unit compares the pressure input from these sections to calculate the thoraco-lumbar spine section position and movement. For instance, if the value of ' $a$ ' is much greater than ' $b$ ', ' $c$ ' and ' $d$ ' and both ' $e$ ' and ' $f$ ' are 0 , that means the user is heavily leaning to the left-front direction. However, if the value of ' $e$ ' and ' $f$ ' are also great at same time, that means the user is in a left leaning 'sloppy' position, as the thoracic area is positioned backwards.

\section{Advisor Unit}

Based on the posture recognition, chair-id and profile, the advisor service calculates a suitability score for each advice in the activity database. Advice is generated through an expert system that considers the following factors:

- Spine angle: sharp angles cause extremely heavy burden on the spine and should not be maintained for a prolonged time

- Prolonged time: this parameter measures the duration of a position

- Accumulated sitting time: cumulative sitting time on different chairs to calculate total sitting time

- Frequency: how often the user takes same position

Instead of monitoring how the user follows the suggested activities, this system follows a decide-choose-do format to accumulate the chosen activities into the database. The "Advisor Unit" sends a query to one or more of the services and acquires their analysis results. If all the factors are met, the advice becomes a candidate. The advices mainly contain several types of message:

- Warning: to alert the user it's time to change posture and stop sitting

- Activity: to suggest users take a proactive relaxation approach and what kind of activity is appropriate for their context. This type of message normally gives user several option to choose from depending on the contextual restrictions and time limitations

- Relaxation: to give the option to rest rather than doing exercises; this type of message suggests a minimum time-span during which any sitting should be avoided

A sample scenario: Mr. A has been continuously working in his office chair for more than 3 hours, and only maintained a healthy posture for less than $20 \%$ of this period. He will receive the following message "Please stand up straight with your arms at your sides, bend sideways to the left, slide your left hand down your thigh and reach with your right arm over your head. Hold this position for 10 seconds, then return to the starting position and repeat for the opposite side. Alternate sides for 9 more times".

\section{Interactive $3 D$ Unit}

The 3D display unit presents spine information in two modes: "real-time mode" and "accumulation mode". The real-time mode presents the user's current spinal position and corresponding burden. The accumulation mode presents the cumulative spinal burden information gathered during a certain period. Advice is mainly generated based on the accumulation mode data. It's very rare that a warning advice is directly generated from the real-time mode as this only happens when a user's movement results in an extreme burden to the spine.

\section{E. Messaging Unit}

Unlike conventional location-based pervasive functions, the Messaging Unit application uses the "chair-id" to recognize users' locations. This unit interacts with users by sending messages generated by the Advisory unit through the most appropriate medium.

\section{EVALUATION TEST SETTING}

In order to evaluate the "Virtual-spine" system we will conduct a user study in late 2013. The test will be conducted using a working prototype, in a specifically designed studio-like environment (ITIDLab, Monash Univeristy, Australia). In order to simulate realistic sitting postures, the furniture and equipment in the room will be arranged same as a conventional office. Standard office chairs, sofas and dining chairs will be equipped with mats embedded with sensors. 16 subjects in total ( 8 male and 8 female) will participate in the study. Each subject will spend 60 minutes sitting while performing assigned tasks that are relevant to the given context.

The data gathered will be analyzed to answer questions such as: How long can the subjects remain in a correct posture 
(without reminding)? How often subjects change sitting positions? Is there any extreme voluntary spinal movement during such a short period? If so, how often? How many alerts subjects receive during the testing period? How do subjects react to the alerts?

\section{CONCLUSIONS}

In this paper we describe the design, progressive implementation and evaluation test plan of a system called Virtual-spine. The combination of direct-sensory input, interactive $3 \mathrm{D}$ representation and contextual advice provides a simple but sufficient method to achieve intuitive sitting support. The advice unit employs an algorithm that takes into account users' profile as well as multiple sedentary information inputs to generate contextual advice. The application can be beneficial for people who want to maintain spinal health by adopting healthy sitting habits, especially for those who seldom exercise and spend most of their time in prolonged sedentary positions.

The planned evaluation test will provide data to better understand usage patterns and reactions to the application. Furthermore, it will indicate whether Virtual-spine is effective in changing behavior. Moving forward beyond the coming evaluation process, our aims are to extend our simulation work to create mixed reality distributed shared spaces with a richer combination of simulated spine movements and applications. We will also make the user interface more intuitive and the advice more personalized based on user feedback.

\section{REFERENCES}

[1] A. Bauman, et al., "The descriptive epidemiology of sitting: a 20-country comparison using the international physical activity questionnaire (IPAQ)," American Journal of Preventive Medicine, vol. 41(2), pp. 228235, 2011.

[2] D. Roffey, et al., "Causal assessment of occupational sitting and low back pain: results of a systematic review," The Spine Journal, vol. 10(3), pp. 252-261, 2010.

[3] Y. Brink and Q. A. Louw, "A systematic review of the relationship between sitting and upper quadrant musculoskeletal pain in children and adolescents". Manual Therapy, in Press.

[4] P. Paoli and D. Merllié, "Third European survey on working conditions 2000," European Foundation for the Improvement of Living and Working Conditions, Luxembourg, Loughlinstown, Ireland, 2001.

[5] M. van Tulder, B. Koes, and L. Bouter, "A cost-of-illness study of back pain in The Netherlands," Pain, vol. 62(2), pp. 233-240, Aug. 1995.

[6] D. Cohen, "An objective measure of seat comfort," Aviation, Space, and Environmental Medicine, vol. 69(4), pp. 410-4, Apr. 1998.

[7] M. G. Halender and L. Zhang, "Field studies of comfort and discomfort in sitting," Ergonomics, vol. 40(9), pp. 895-915, Sep. 1997.

[8] M. Monette, R. Weiss-Lambrou, and J. Dansereau. "In search of a better understanding of wheelchair sitting comfort and discomfort," in RESNA Annual Conference, 1999.

[9] G. Shaw, "Wheelchair seat comfort for the institutionalized elderly," Assistive Technology, vol. 3(1), pp. 11-23, 1992.

[10] "Pressure ulcers in adults: Prediction and prevention, clinical practice guideline," Agency for Health Care Policy \& Research (AHCPR), AHCPR Publication no. 92-0047, 1992.
[11] G. H. Brandeis, et al., "A longitudinal study of risk factors associated with the formation of pressure ulcers in nursing homes," Journal of American Geriatric Society, vol. 42(4), pp. 388-93, 1994.

[12] D. M. Smith, "Pressure ulcers in the nursing home," Annals of Internal Medicine, vol. 123(6), pp. 433-438, Sept. 1995.

[13] J. Caneiro, et al., "The influence of different sitting postures on head/neck posture and muscle activity," Manual Therapy, vol. 50(1), pp. 54-60, Feb. 2010.

[14] A. Burnett, et al., "Spinal kinematics and trunk muscle activity in cyclists: a comparison between healthy controls and non-specific chronic low back pain subjects," Manual Therapy, vol. 9, pp. 211-219, 2004.

[15] K. O'Sullivan, et al., "What do physiotherapists consider to be the best sitting spinal posture?," Manual Therapy, vol. 17(5), pp. 432-7, Oct. 2012.

[16] M. Pope, K. Goh, and M. Magnusson, "Spine ergonomics," Annual review of biomedical engineering, vol. 4(1), pp. 49-68, 2002.

[17] A. P. Claus, et al., "Is 'ideal' sitting posture real?: Measurement of spinal curves in four sitting postures," Manual Therapy, vol. 14(4), pp. 404-408, 2009.

[18] M. Haller, et al. "Finding the right way for interrupting people improving their sitting posture," in INTERACT'11 Proceedings of the 13th IFIP TC 13 international conference on Human-computer interaction. 2011.

[19] M. Davies, V. Callaghan, and L. Shen, "Modelling pervasive environments using bespoke and commercial game-based simulators," Lecture Notes in Computer Science, vol. 4689, pp. 67-77, 2007.

[20] M. Billinghurst and H. Kato. "Collaborative mixed reality," in Proc. Int'1 Symp. Mixed Reality (ISMR 99). Secaucus, N.J.: Springer-Verlag, 1999.

[21] V. Callaghan, et al., "Intelligent inhabited environments," BT Technology Journal, vol. 22(3), pp. 233-247, 2004.

[22] R. Azuma, et al., "Recent advances in augmented reality," Computer Graphics and Applications, IEEE, vol. 21(6), pp. 34 - 47, 2001.

[23] B. J. Fogg and D. Eckles, eds, Mobile Persuasion: 20 Perspective On The Future Of Behavior Change. California: Stanford Captology Media, 2007.

[24] B. J. Fogg, Persuasive Technology: Using Computers to Change What We Think and Do. San Francisco, CA: Morgan Kaufmann. 2003.

[25] P. Boland, "Managing chronic diserse through mobile persuasion", in Early Movers, B.J. Fogg and D. Eckles, eds, Mobile Perusasion: 20 Perspective on the Future of Behavior Change. California: Stanford Captology Media, 2007, pp. 39-45.

[26] S. S. Intille, "A new research challenge: Persuasive technology to motivate healthy aging," IEEE Transactions on Information Technology in Biomedicine, vol. 8(3), pp. 235-237, Sep. 2004.

[27] S. S. Intille, "Ubiquitous computing technology for just-in-time motivation of behavior chang," Studies in Health Technology and Informatics, vol. 107(Pt 2), pp. 1434-1437, 2004.

[28] M. Maheshwari, S. Chatterjee, and D. Drew. "Exploring the persuasiveness of "Just-in-time" motivational messages for obesity management," in PERSUASIVE '08 Proceedings of the 3rd International Conference on Persuasive Technology. Berlin, Heidelberg: SpringerVerlag, 2008.

[29] L. Frank, P. Engelke, and S. T., Health and Community Design: The Impact of the Built Environment on Physical Activity. Washington, DC: Island Press, 2003.

[30] B. Mutlu, et al. "Robust, lowcost, nonintrusive sensing and recognition of seated postures," in UIST '07 Proceedings of the 20th Annual ACM Symposium on User Interface Software and Technology. New York, NY: ACM, 2007.

[31] "Project: unity-arduino with serial connection", [online] Available: http://code.google.com/p/unity-arduino-serial-connection/, [Accessed: 27 Feb. 2013]. 